\section{Algumas repercussões emocionais negativas da gravidez precoce em adolescentes do Município do Rio de Janeiro (1999-2001)}

\author{
Some emotional repercussions of adolescent \\ pregnancy in Rio de Janeiro, Brazil (1999-2001)
}

\author{
${ }^{1}$ Departamento de \\ Epidemiologia e Métodos \\ Quantitativos em Saúde, \\ Escola Nacional \\ de Saúde Pública, \\ Fundação Oswaldo Cruz, \\ Rio de Janeiro, Brasil. \\ Correspondência \\ Maria do Carmo Leal \\ Rua Leopoldo Bulhões 1480, \\ Rio de Janeiro, $R J$ \\ 21041-210, Brasil. \\ duca@ensp.fiocruz.br
}

\begin{abstract}
This study analyzed the association between early pregnancy and negative emotional repercussions for adolescents, according to socio-demographic and psychosocial variables. The study interviewed 1,228 adolescents in maternity hospitals in the City of Rio de Janeiro. Emotional repercussions were evaluated with negative self-assessment variables, showing little or no expectation towards the future, as well as psychological distress. The $\chi^{2}$ test and odds ratio were used to analyze the data. Adolescents who reported a negative reaction by the family felt less valued (OR 1.8), with little expectation towards the future (OR 2.3), and presented major psychological distress (OR 1.5). Those not enrolled in school when they became pregnant also showed worse self-esteem (OR 1.9) and fewer expectations towards the future (OR 2.2). Greater psychological distress was inversely related to frequency of prenatal care (0.7). The article concludes that family support, regardless of original social conditions, was identified as the principal factor for minimizing negative emotional repercussions of pregnancy during adolescence.

Pregnancy in Adolescence; Psychological Stress; Adolescent
\end{abstract}

\author{
Adriane Reis Sabroza 1 \\ Maria do Carmo Leal 1 \\ Paulo Roberto de Souza Jr. 1 \\ Silvana Granado Nogueira da Gama 1
}

\section{Introdução}

A complexidade característica da gravidez precoce é influenciada por diferenças sociais, culturais e econômicas da adolescente que a vivencia. O fato de a adolescente vir a ultrapassar etapas importantes, do ponto de vista do desenvolvimento, em função de uma gestação nesse período, constitui-se como uma experiência emocionalmente difícil.

A responsabilidade precoce imposta pela gravidez, paralela a um processo de amadurecimento, ainda em curso, resulta em uma adolescente mal preparada para assumir as responsabilidades psicológicas, sociais e econômicas que a maternidade envolve 1,2 . A instabilidade das relações conjugais também acaba por contribuir para a ocorrência de prejuízos emocionais e até mesmo de transtornos de ordem afetiva 3,4 , muitas vezes agravados por um ambiente familiar pouco acolhedor e muito mobilizado pela notícia da gestação. Alguns estudos também mostram que o risco dos filhos serem vítimas de maus-tratos é maior 5 , especialmente nos casos em que a gravidez foi indesejada 6 .

Nesse sentido, alguns aspectos psicológicos, tais como baixa auto-estima, ausência de apoio familiar, vivência de alto nível de estresse, poucas expectativas frente ao futuro e a presença de sintomas depressivos, além de comuns, exercem influência no modo que a rela- 
ção entre a gestante adolescente e seu bebê irá se constituir. De acordo com Barnet et al. 7, a prevalência de sintomas depressivos durante a gestação pode variar entre 44,0 e 59,0\%, sendo comuns em gestantes adolescentes.

Diante da possibilidade de tantas conseqüências psicológicas adversas, torna-se relevante o estudo de aspectos emocionais da gravidez precoce. Nesse sentido, o objetivo do presente trabalho é verificar a associação entre gravidez precoce e as repercussões emocionais negativas na vida da adolescente, com o intuito de se pensar novas formas de assistência que sejam capazes de minimizar os prejuízos emocionais que uma gestação pode levar à vida das adolescentes, principalmente quando não desejada.

\section{Material e método}

Este estudo foi desenvolvido dentro da linha de investigação sobre A Gravidez na Adolescência como Variável Explicativa do Baixo Peso ao Nascer e Outros Efeitos Adversos no Recém-Nascido em Maternidades do Município do Rio de Janeiro, realizada em uma amostra de puérperas que se hospitalizaram para o parto em maternidades localizadas no município.

\section{Amostra}

Trata-se de um estudo descritivo do tipo transversal com uma amostra de 1.228 puérperas de 12 a 19 anos de idade. Este número refere-se a uma subamostra do "Estudo da Morbi-mortalidade e da Atenção Peri e Neonatal no Município do Rio de Janeiro", que selecionou, aleatoriamente, uma amostra estratificada proporcional de $10 \%$ dos partos dentre os três estratos formados: estabelecimentos municipais e federais, estrato 1; estabelecimentos militares, estaduais, filantrópicos e privados conveniados com o SUS, estrato 2; e estabelecimentos privados, estrato 3. Para os três estratos amostrais, selecionou-se, de forma sistemática, duas em cada três gestantes adolescentes, correspondendo assim a $62,3 \%$ do total das adolescentes da amostra total. Maiores detalhes sobre a metodologia do projeto, consultar Leal et al. ${ }^{8}$.

\section{Considerações éticas}

Este estudo foi submetido ao Comitê de Ética para Pesquisa da Escola Nacional de Saúde Pú- blica da Fundação Oswaldo Cruz. A participação da adolescente, bem como a autorização do responsável, em caso de menores de idade, foi obtida por meio de termo de consentimento livre e esclarecido por escrito, no qual era exposto o objetivo da pesquisa. A confidencialidade da informação quanto ao conteúdo e à identificação da puérpera, assim como o anonimato por ocasião da publicação dos resultados foram assegurados.

\section{Desenvolvimento do estudo}

Dados sócio-demográficos foram coletados diretamente por meio de um questionário aplicado por acadêmicos bolsistas de enfermagem e medicina previamente treinados. As informações sobre variáveis psicossociais, estilo de vida e repercussões negativas da gestação foram coletadas com base em um segundo questionário, aplicado por uma psicóloga e outras três entrevistadoras da área de saúde, treinadas pela primeira. Todos os questionários foram revisados e codificados antes da digitação.

A partir de um conjunto de variáveis, mostradas no quadro abaixo, construiu-se os indicadores expectativa em relação ao futuro e autovalorização. A adolescente respondia o questionário indicando o seu grau de concordância com as afirmativas feitas, numa escala que variava de 0 a 3, sendo 0 para discordância e 3 para total concordância. O somatório das notas que a adolescente se atribuía, dividido pelo número de perguntas, compunha a sua média individual naquele indicador. Antes de fazer o somatório para tirar a média da adolescente, as respostas negativas foram computadas no sentido inverso, ou seja, valor 3 para discordância e valor 0 para concordância, permitindo deste modo que todas as variáveis ficassem na mesma direção. O ponto de corte estipulado para os indicadores expectativas em relação ao futuro e autovalorização foi estabelecido em 1,4, abaixo do qual, as expectativas eram consideradas poucas e negativas. Estes pontos de corte foram discriminados pelo quartil inferior da distribuição (Tabela 1).

Para o indicador nível de sofrimento psíqui$c o$, as opções de resposta eram: "com freqüência", "raramente" e "nunca", sendo 2, 1 e 0 os valores atribuídos respectivamente a cada resposta. $\mathrm{O}$ indicador foi construído com base na média do somatório dos escores atribuídos a cada item. O ponto de corte estabelecido foi de 0,83 , equivalente ao quartil superior da distri- 
Variáveis que compõem os indicadores de repercussões emocionais negativas.

\begin{tabular}{|c|c|}
\hline Indicadores & Variáveis \\
\hline \multirow[t]{5}{*}{ Expectativa em relação ao futuro } & "Eu tenho planos para o futuro" \\
\hline & "Às vezes penso que nada tenho a esperar do futuro" \\
\hline & "Eu penso que nunca terei dinheiro suficiente" \\
\hline & "Quando eu for mais velha com certeza terei uma vida boa" \\
\hline & "O fato de engravidar na adolescência dificultará a realização dos meus objetivos" \\
\hline \multirow[t]{6}{*}{ Autovalorização } & "Eu gosto do meu jeito de agir" \\
\hline & "As pessoas geralmente gostam de mim" \\
\hline & "Eu gosto da minha aparência" \\
\hline & "Existem muitas coisas que eu faço bem" \\
\hline & "Às vezes penso que não sou boa o suficiente" \\
\hline & "Desisto facilmente diante das dificuldades" \\
\hline \multirow[t]{12}{*}{ Nível de sofrimento psíquico } & "Eu me senti triste" \\
\hline & "Eu me percebi falando menos que o habitual" \\
\hline & "Senti que minha vida tem sido um fracasso" \\
\hline & "Senti solidão" \\
\hline & "Estive menos disposta para iniciar tarefas" \\
\hline & "Na minha família as pessoas brigam" \\
\hline & "Senti que estava deprimida" \\
\hline & "Senti as pessoas menos amigas e mais afastadas" \\
\hline & "Senti que não poderia sair da tristeza mesmo com a ajuda de familiares e amigos" \\
\hline & "Senti vontade de permanecer o tempo todo deitada" \\
\hline & "Pensei em cometer suicídio" \\
\hline & "Fiz alguma tentativa de cometer suicídio" \\
\hline
\end{tabular}

buição, a partir do qual o nível de sofrimento psíquico era considerado grande. Os indicadores, bem como a metodologia utilizada na criação destas variáveis foram baseados em Springer \& Phillips 9 .

As variáveis sociodemográficas utilizadas na análise foram: "cor da pele" (branca/preta ou parda); "estudava ao engravidar" (sim/não) e "vive com o pai do bebê" (sim/não). As variáveis psicológicas e sociais foram: "reação familiar à gestação" (boa/ruim), "tentou interromper a gestação" (sim/não), "fumou na gestação" ( $\mathrm{sim} /$ não), "realizou pré-natal” (até 6 consultas/7 ou mais) e "desejou a gestação" (sim/não).

\section{Análise estatística}

Análises bivariadas foram feitas para exploração inicial dos dados, utilizando-se o teste de $\chi^{2}$ para testar a homogeneidade das proporções, em um nível de significância de 5,0\%. Utilizouse um modelo de regressão logística, método stepwise, baseado na razão de verossimilhança, para cálculo das odds ratio (OR) ajustadas. O ní- vel de significância para inclusão de variáveis no modelo foi estabelecido em $5,0 \%$ e para exclusão em 10,0\%. O teste de Hosmer-Lemeshow foi usado para avaliar o ajuste do modelo.

\section{Resultados}

Na Tabela 2, verifica-se que $23,7 \%$ das puérperas apresentaram uma autovalorização negativa. Houve uma maior proporção de puérperas com autovalorização negativa em famílias que reagiram mal à gestação, 31,0\%, bem como entre as adolescentes que se encontravam fora da escola ao engravidar, $27,1 \%$. Uma maior proporção de puérperas nesta situação foi também encontrada entre as que tentaram interromper a gestação. A faixa etária, a cor da pele, o hábito de fumar durante a gravidez, o número de consultas pré-natais realizadas e o desejo em relação à gestação não apontaram diferenças estatisticamente significativas.

Na Tabela 3, foi possível verificar uma associação estatisticamente significativa entre a cor 
da pele negra ou parda e a presença de pouca ou nenhuma expectativa em relação ao futuro. A reação familiar à gestação também fez diferença, ficando as puérperas cuja reação familiar à gestação foi ruim, em situação de desvantagem, 33,0\%.

Interessante notar que não estar estudando ao engravidar e realizar número insuficiente de consultas pré-natais estiveram correlacionados à pouca ou nenhuma expectativa em relação ao futuro. O desejo quanto à gestação novamente mostra-se relevante, sendo maior a proporção de puérperas com pouca ou nenhuma expectativa em relação ao futuro entre as que desejaram a gestação, 28,8\%, em comparação com as que não desejaram, 18,5\%.

Foi constatado que o nível de sofrimento psíquico foi mais intenso em $26,8 \%$ das adolescentes, sendo estas as que apresentavam características sócio-demográficas e psicossociais menos favoráveis (Tabela 4). Observou-se que não houve diferenças significativas entre puérperas segundo a faixa etária, estar estudando no momento que engravidaram e ter fumado na gestação. Contudo, o nível de sofrimento psíquico foi maior nas adolescentes cuja reação familiar à gestação foi ruim, fizeram poucas ou nenhuma consulta pré-natal, não viviam com o pai do bebê e de cor da pele negra ou parda. O mesmo ocorreu para as que tentaram abortar e afirmaram não ter desejado a gestação, 30,4\% contra 20,6\% das que desejaram.

A Tabela 5 expõe os resultados da análise de regressão logística múltipla. Para a variável autovalorização negativa somente três variáveis independentes foram selecionadas para a inclusão no modelo, de acordo com os critérios estatísticos previamente estabelecidos. "Não estudava quando engravidou”, foi a variável que mais explicou, com uma razão de produtos cruzados (OR) ajustada 1,96 ( $\mathrm{p}=0,004)$. As outras duas foram a "reação familiar ruim" com uma OR ajustada igual a 1,78 ( $\mathrm{p}=0,009)$ e "tentou interromper a gestação”, cuja OR ajustada foi 0,46 ( $p=0,006$ ), apresentando-se como um efeito protetor para a autovalorização negativa.

Com relação à pouca ou nenhuma expectativa em relação ao futuro, três variáveis permaneceram no modelo. A que mais explicou este desfecho foi "não estudava quando engravidou”, OR ajustada 2,16 ( $\mathrm{p}=0,000)$, seguida pelas variáveis "reação familiar ruim", OR 2,28 ajustada ( $p=0,000)$ e "não desejou a gestação", OR ajustada 1,94 ( $\mathrm{p}=0,016)$.

O maior sofrimento psíquico foi explicado por três variáveis: "reação familiar ruim”, OR ajustado 1,51 ( $\mathrm{p}=0,043)$, "tentou interromper a gestação", OR ajustada 2,09 (p = 0,002) e "nú-

\section{Tabela 2}

Porcentagem de puérperas com autovalorização negativa, segundo variáveis sócio-demográficas e psicossociais. Município do Rio de Janeiro, Brasil, 1999-2001.

\begin{tabular}{|c|c|c|c|c|}
\hline \multirow[t]{2}{*}{ Variáveis } & \multicolumn{4}{|c|}{ Autovalorização negativa } \\
\hline & $\mathrm{n}$ & $\%$ & $\chi_{2}$ & $p$ valor \\
\hline \multicolumn{5}{|l|}{ Faixa etária (anos) } \\
\hline $12-16$ & 72 & 22,8 & & \\
\hline 17-19 & 215 & 24,0 & 0,198 & 0,701 \\
\hline \multicolumn{5}{|l|}{ Cor } \\
\hline Branca & 127 & 24,9 & 0,705 & 0,412 \\
\hline Parda/negra & 160 & 22,8 & & \\
\hline \multicolumn{5}{|l|}{ Vive com o pai do bebê } \\
\hline $\operatorname{Sim}$ & 209 & 25,3 & 3,693 & 0,059 \\
\hline Não & 78 & 20,3 & & \\
\hline \multicolumn{5}{|l|}{ Estudava ao engravidar } \\
\hline Sim & 112 & 19,8 & 8,718 & 0,004 \\
\hline Não & 174 & 27,1 & & \\
\hline \multicolumn{5}{|l|}{ Reação familiar à gestação } \\
\hline Boa & 175 & 20,6 & 15,263 & 0,000 \\
\hline Ruim & 112 & 31,0 & & \\
\hline \multicolumn{5}{|c|}{ Tentou interromper a gestação } \\
\hline Sim & 21 & 18,9 & 6,326 & 0,012 \\
\hline Não & 113 & 31,2 & & \\
\hline \multicolumn{5}{|l|}{ Fumou na gestação } \\
\hline Sim & 28 & 21,5 & 0,383 & 0,586 \\
\hline Não & 259 & 24,0 & & \\
\hline \multicolumn{5}{|c|}{ Número de consultas pré-natal } \\
\hline 0 a 3 & 48 & 27,9 & 5,638 & 0,060 \\
\hline 4 a 6 & 87 & 20,2 & & \\
\hline 7 ou mais & 137 & 25,7 & & \\
\hline \multicolumn{5}{|l|}{ Desejou a gestação } \\
\hline Sim & 101 & 22,8 & 0,284 & 0,594 \\
\hline Não & 185 & 24,2 & & \\
\hline Número total da amostra & 287 & 23,7 & 1.228 & \\
\hline
\end{tabular}


Tabela 3

Porcentagem de puérperas com pouca ou nenhuma expectativa em relação ao futuro, segundo variáveis sócio-demográficas e psicossociais. Município do Rio de Janeiro, Brasil, 1999-2001.

\begin{tabular}{|c|c|c|c|c|}
\hline \multirow[t]{2}{*}{ Variáveis } & \multicolumn{4}{|c|}{$\begin{array}{l}\text { Pouca ou nenhuma expectativa } \\
\text { em relação ao futuro }\end{array}$} \\
\hline & $\mathrm{n}$ & $\%$ & $\chi^{2}$ & $p$ valor \\
\hline \multicolumn{5}{|l|}{ Faixa etária (anos) } \\
\hline $12-16$ & 85 & 26,9 & 0,804 & 0,365 \\
\hline $17-19$ & 218 & 24,3 & & \\
\hline \multicolumn{5}{|l|}{ Cor } \\
\hline Branca & 110 & 21,4 & 6,096 & 0,016 \\
\hline Parda/negra & 193 & 27,6 & & \\
\hline \multicolumn{5}{|l|}{ Vive com o pai do bebê } \\
\hline Sim & 200 & 24,1 & 1,147 & 0,285 \\
\hline Não & 103 & 27,0 & & \\
\hline \multicolumn{5}{|l|}{ Estudava ao engravidar } \\
\hline $\operatorname{Sim}$ & 117 & 20,7 & 10,390 & 0,001 \\
\hline Não & 186 & 28,8 & & \\
\hline \multicolumn{5}{|l|}{ Reação familiar à gestação } \\
\hline Boa & 184 & 21,6 & 17,485 & 0,000 \\
\hline Ruim & 119 & 33,0 & & \\
\hline \multicolumn{5}{|c|}{ Tentou interromper a gestação } \\
\hline Sim & 44 & 38,9 & 3,345 & 0,083 \\
\hline Não & 108 & 29,8 & & \\
\hline \multicolumn{5}{|l|}{ Fumou na gestação } \\
\hline Sim & 34 & 26,0 & 0,071 & 0,831 \\
\hline Não & 269 & 24,9 & & \\
\hline \multicolumn{5}{|c|}{ Número de consultas pré-natal } \\
\hline $0-3$ & 59 & 34,1 & & \\
\hline $4-6$ & 115 & 26,6 & 10,728 & 0,005 \\
\hline 7 ou mais & 116 & 21,8 & & \\
\hline \multicolumn{5}{|l|}{ Desejou a gestação } \\
\hline $\operatorname{Sim}$ & 82 & 28,8 & 15,782 & 0,000 \\
\hline Não & 221 & 18,5 & & \\
\hline Número total da amostra & 303 & 25,0 & & \\
\hline
\end{tabular}

mero de consultas pré-natal", OR ajustada 0,75 ( $\mathrm{p}=0,033$ ). Esta última, apresentando-se como um efeito protetor para o sofrimento psíquico intenso.

\section{Discussão}

O estudo de repercussões emocionais negativas de uma gestação precoce em adolescentes que, em geral, encontram-se despreparadas para lidar com a nova situação da maternidade, é tema atual da literatura 10,11.

Os resultados do presente estudo evidenciam que são intensas as repercussões emocionais da gestação em puérperas adolescentes. Os dados apontam para um quadro desfavorável 12, em que muitas adolescentes gestantes apresentam uma autovalorização negativa e baixa expectativa em relação ao futuro.

Um grande sofrimento psíquico esteve presente em quase um terço dos casos, chamando a atenção por se tratar de adolescentes com nível realmente alto de estresse emocional.

Alguns autores referem uma baixa autovalorização como característica de adolescentes que têm relações sexuais e não utilizam métodos contraceptivos 13, o que ocorre mais freqüentemente nas camadas mais pobres da população.

Foi menor a proporção de adolescentes que manifestaram ter desejado esta gravidez entre as que apresentaram um grande sofrimento psíquico. De modo contrário, observamos que foi maior a proporção de adolescentes que desejaram a gestação entre as que apresentaram pouca ou nenhuma expectativa em relação ao futuro. Como verificado em outros estudos 14 , esse fato leva a pensar que a gravidez precoce representaria efetivamente uma possibilidade de reconhecimento e/ou status social, almejado por adolescentes cujas vidas foram capazes de lhes apresentar tão poucas oportunidades. A gravidez acabaria por significar uma tentativa de reconhecimento social ou uma resposta adaptativa à situação de pobreza, daí uma menor prevalência de sofrimento psíquico nestes casos 15.

A cor da pele, variável muito analisada na literatura internacional e, de um modo geral, descrita como relacionada à condição socioeconômica, também apontou algumas associações interessantes com os indicadores aqui utilizados. Enquanto evidenciou-se uma maior proporção de adolescentes de cor negra/parda entre as com pouca ou nenhuma expectativa em relação ao futuro, obteve-se uma maior proporção de brancas com uma autovalorização 
negativa. Nos dois casos, a condição social da adolescente parece exercer papel importante na associação encontrada, só que de maneira diversa. Para a adolescente de cor negra/parda, as expectativas em relação ao futuro encontram-se bastante reduzidas, confirmando um lugar de desvalorização na escala social em função da raça. Por outro lado, a presença de maior autovalorização negativa entre as brancas, geralmente mais valorizadas socialmente, pode significar uma característica de personalidade que inclusive pode ter contribuído para a ocorrência desta gestação.

A aderência às consultas do atendimento pré-natal foi também influenciada por fatores emocionais, neste estudo. Outros fatores, tais como influência familiar, facilidade de transporte e possibilidades econômicas, foram identificados por Lee \& Grubbs 16 como facilitadores da aderência ao atendimento pré-natal.

O consumo de cigarro somente esteve associado ao nível de sofrimento psíquico, o que corrobora com outros estudos em que se verificou que adolescentes têm motivações psicossociais específicas e diferentes dos adultos para o consumo de cigarros 17 .

Do mesmo modo, a reação familiar ruim, esteve associada a um grande sofrimento psíquico, apontando a importância do apoio social para uma melhor vivência da gestação pela adolescente 18 e, conseqüentemente, para um desenvolvimento gestacional adequado. Este fato se confirma ao observarmos os dados referentes às adolescentes que apresentaram poucas expectativas em relação ao futuro e uma autovalorização negativa.

Os resultados da regressão logística indicaram que estar fora da escola quando engravidou, assim como, ter uma reação familiar ruim, contribuíram para uma autovalorização negativa da adolescente. Este fato é condizente com achados da literatura, uma vez que a relação familiar e o nível de escolaridade são referidos como importantes fatores da constituição do autoconceito de um indivíduo ${ }^{19}$. A pouca ou nenhuma expectativa em relação ao futuro também foi parcialmente explicada por não estar estudando, reação familiar ruim e não ter desejado a gestação, revelando que o aspecto familiar e a motivação para a gravidez constituem importantes influências no modo como a adolescente lida com as expectativas de futuro e que pode comprometer inclusive a relação mãebebê.

Diante do exposto, reconhece-se não apenas a importância que o presente estudo traz, no sentido de uma maior compreensão sobre aspectos emocionais de uma gravidez precoce,
Tabela 4

Porcentagem de puérperas com sofrimento psíquico grande, segundo variáveis sócio-demográficas e psicossociais. Município do Rio de Janeiro, Brasil, 1999-2001.

\begin{tabular}{|c|c|c|c|c|}
\hline \multirow[t]{2}{*}{ Variáveis } & \multicolumn{4}{|c|}{ Sofrimento psíquico intenso } \\
\hline & $\mathrm{n}$ & $\%$ & $\chi^{2}$ & $p$ valor \\
\hline \multicolumn{5}{|l|}{ Faixa etária (anos) } \\
\hline $12-16$ & 97 & 30,5 & 3,086 & 0,089 \\
\hline $17-19$ & 226 & 25,4 & & \\
\hline \multicolumn{5}{|l|}{ Cor } \\
\hline Branca & 111 & 21,6 & 11,948 & 0,001 \\
\hline Parda/negra & 212 & 30,5 & & \\
\hline \multicolumn{5}{|l|}{ Vive com o pai do bebê } \\
\hline Sim & 186 & 22,6 & 23,118 & 0,000 \\
\hline Não & 137 & 35,8 & & \\
\hline \multicolumn{5}{|l|}{ Estudava ao engravidar } \\
\hline Sim & 163 & 29,0 & 2,432 & 0,134 \\
\hline Não & 160 & 25,0 & & \\
\hline \multicolumn{5}{|l|}{ Reação familiar à gestação } \\
\hline Boa & 195 & 23,0 & 20,624 & 0,000 \\
\hline Ruim & 128 & 35,7 & & \\
\hline \multicolumn{5}{|c|}{ Tentou interromper a gestação } \\
\hline Sim & 54 & 48,6 & 12,789 & 0,001 \\
\hline Não & 109 & 30,2 & & \\
\hline \multicolumn{5}{|l|}{ Fumou na gestação } \\
\hline Sim & 41 & 31,5 & 1,681 & 0,208 \\
\hline Não & 282 & 26,2 & & \\
\hline \multicolumn{5}{|c|}{ Número de consultas pré-natal } \\
\hline $0-3$ & 61 & 35,9 & 8,216 & 0,016 \\
\hline $4-6$ & 120 & 27,8 & & \\
\hline 7 ou mais & 131 & 24,6 & & \\
\hline \multicolumn{5}{|l|}{ Desejou a gestação } \\
\hline Sim & 91 & 20,6 & 13,750 & 0,000 \\
\hline Não & 232 & 30,4 & & \\
\hline Número total da amostra & 323 & 26,8 & & \\
\hline
\end{tabular}


Resultados das Regressões Logísticas Múltiplas das variáveis dependentes: autovalorização negativa, expectativa em relação ao futuro e sofrimento psíquico intenso. Município do Rio de Janeiro, Brasil, 1999-2001.

\begin{tabular}{|c|c|c|c|}
\hline Variáveis & OR ajustado & $\mathrm{p}$ valor & IC95\% \\
\hline \multicolumn{4}{|l|}{ Autovalorização negativa* } \\
\hline Não estudava quando engravidou & 1,923 & 0,004 & $1,233-2,998$ \\
\hline Reação familiar ruim & 1,781 & 0,009 & $1,153-2,751$ \\
\hline Tentou interromper a gestação & 0,458 & 0,006 & $0,261-0,803$ \\
\hline \multicolumn{4}{|c|}{ Pouca ou nenhuma expectativa em relação ao futuro ${ }^{\star \star}$} \\
\hline Não Estudava quando engravidou & 2,157 & 0,000 & $1,419-3,280$ \\
\hline Reação familiar ruim & 2,280 & 0,000 & $1,508-3,448$ \\
\hline Não desejou a gestação & 1,938 & 0,016 & $1,130-3,323$ \\
\hline \multicolumn{4}{|l|}{ Sofrimento psíquico intenso ${ }^{\star \star *}$} \\
\hline Reação familiar ruim & 1,512 & 0,043 & $1,012-2,259$ \\
\hline Tentou interromper a gestação & 2,088 & 0,002 & $1,315-3,314$ \\
\hline Pré-natal & 0,746 & 0,033 & $0,570-0,977$ \\
\hline
\end{tabular}

* Teste de Hosmer-Lemeshow $p=0,55$

$\star * *$ Teste de Hosmer-Lemeshow $p=0,19$

*** Teste de Hosmer-Lemeshow $p=0,69$.

como também se afirma a necessidade de uma visão multidisciplinar do fenômeno.

Profissionais habilitados ao manejo de aspectos emocionais devem ser incluídos na formulação de políticas públicas, que visem tanto a prevenção da gestação em adolescentes, quanto o atendimento desta população específica na rede assistencial, visando, desse modo, a minimizar as conseqüências emocionais negativas da gravidez precoce.
Um aspecto a destacar na metodologia utilizada neste estudo é que os resultados analisados tomaram como ponto de corte o quartil da distribuição da freqüência das variáveis, resultando na comparação das $25,0 \%$ que tinham piores resultados com as outras. Outros pontos de corte podem encontrar resultados distintos deste apresentado.

\section{Resumo}

Analisou-se a associação entre gravidez precoce e as repercussões emocionais negativas para a adolescente, segundo variáveis sócio-demográficas e psicossociais. Foram entrevistadas 1.228 adolescentes em maternidades do Município do Rio de Janeiro. Avaliou-se as repercussões emocionais por meio das variáveis autovalorização negativa, pouca ou nenhuma expectativa frente ao futuro e sofrimento psíquico. O teste do $\chi^{2}$ e a Odds Ratio foram usadas para análise dos dados. As adolescentes que referiram uma reação negativa da família se sentiram menos valorizadas $(O R 1,8)$, com poucas expectativas em relação ao futuro $(\mathrm{OR} 2,3) \mathrm{e}$ manifestaram grande sofrimento psíquico $(O R$ 1,5). As que estavam fora da escola quando engravidaram tiveram também uma pior autovalorização $(O R 1,9) e$ menores expectativas em relação ao futuro $(O R 2,2)$. Um grande nível de sofrimento psíquico relacionou-se inversamente à freqüência ao pré-natal $(0,7)$. Pode-se concluir que o suporte familiar, independente da condição social de origem, foi identificado neste estudo como o principal fator minimizador das repercussões emocionais negativas da gestação na adolescência.

Gravidez na Adolescência; Estresse Psicológico; Adolescente 


\section{Colaboradores}

A. R. Sabroza estabeleceu os tópicos abordados no estudo, aprofundou a discussão na área de psicologia e redigiu o artigo. M. C. Leal contribuiu na análise dos dados, descrição dos resultados, discussão e revisão final do texto. P. R. B. Souza Jr. colaborou na análise estatística dos resultados e na elaboração da metodologia empregada no artigo. S. G. N. Gama contribuiu na definição do objeto de estudo, análise dos dados e na redação do artigo.

\section{Referências}

1. Silva MCSC. Hábitos e comportamento do adolescente. Curitiba: Universidade Federal do Paraná; 1987.

2. Motta M, Pinto-Silva JL. Gravidez na adolescência. Campinas: Núcleo de Estudos de População, Universidade Estadual de Campinas; 1994.

3. Montessoro AC, Blixen CE. Public policy and adolescent pregnancy: a reexamination of the issues. Nurs Outlook 1996; 44:31-6.

4. Perrin KM, McDermott RJ. Instruments to measure social support and related constructs in pregnancy adolescents: a review. Adolescence 1997; 22:533-57.

5. Conger JJ, Petersen A. Adolescence and youth: psychological development in a changing world. New York: Harper \& Row; 1984.

6. Azevedo MA, Guerra VA. Crianças vitimizadas: a síndrome do pequeno poder. São Paulo: Editora Iglu; 1985.

7. Barnet B, Joffe A, Duggan AK, Wilson MD, Reepke JT. Depressive symptoms, stress and postpartum adolescents. Arch Pediatr Adolesc Med 1996; 1150:64-9.

8. Leal MC, Gama SGN, Campos MR, Cavalini LT, Garbayo LS, Brasil CLP, et al. Fatores associados à morbi-mortalidade perinatal em uma amostra de maternidades públicas e privadas do Município do Rio de Janeiro, 1999-2001. Cad Saúde Pública 2004; 20 Suppl 1:S20-33.

9. Springer JF, Phillips JL. Individual Protective Factors Index (IPFI): a measure of adolescent resilience. Folson: EMT Associates Inc.; 1997.

10. Cabral ACV, Peixoto RML, Miranda SP, Vieira E. Gravidez e adolescência. J Bras Ginecol 1985; 95: 251-3.

11. Skari H, Skreden M, Malt UF, Dalholt M, Ostensen $\mathrm{AB}$, Egeland T, et al. Comparative levels of psychological distress, stress symptoms, depression and anxiety after childbirth - a prospective population-based study of mothers and fathers. $\mathrm{Br} \mathrm{J}$ Obstet Gynaecol 2002; 109:1154-63.
12. Gama SGN, Leal MC, Szwarcwald CL. Experiência de gravidez na adolescência, fatores associados e resultados perinatais entre puérperas de baixa renda. Cad Saúde Pública 2002; 18:153-61.

13. Consejo Nacional de Población. Antología de la sexualidad humana. México, DF: Ilus; 1994.

14. Stevens-Simon C, Low YR. Teenage childbearing. $\mathrm{Na}$ adaptative strategy for the socioeconomic ally disadvantaged or a strategy for adapting to socioeconomic disadvantage? Arch Pediatr Adolesc Med 1995; 149:912-5.

15. Unger JB, Molina GB, Teran L. Perceived consequences of teenage childbearing among adolescent girls in an urban sample. J Adolesc Health 2000; 26:205-12.

16. Lee SH, Grubbs LM. A comparison of self-reported self-care practices of pregnant adolescents. Nurse Pract 1993; 18:25-9.

17. Wang MQ, Fitzhugh EC, Green BL, Turner LW, Eddy JM, Westerfield C. Prospective social-psychological factors of adolescent smoking progression. J Adolesc Health 1999; 24:2-9.

18. Dormire RN, Strauss RN, Clarke RN. Social Support and adaptation to the parent role in first-time adolescent mothers. J Obstet Gynecol Neonatal Nurs 1989; 18:327-36.

19. Humprhreys T. Auto-estima: a chave para a educação de seu filho. São Paulo: Ground; 2001.

Recebido em 02/Out/2002

Versão final reapresentada em 08/Out/2003

Aprovado em 28/Out/2003 